\title{
Tendencias de los fenotipos de resistencia bacteriana en hospitales públicos y privados de alta complejidad de Colombia
}

\author{
Andrea Patricia Villalobos Rodríguez, ${ }^{1}$ Miguel Hernando Díaz Ortega, ${ }^{1}$ \\ Liliana Isabel Barrero Garzón, ${ }^{1}$ Sandra Milena Rivera Vargas, ${ }^{1}$ \\ Daibeth Elena Henríquez Iguarán, ${ }^{2}$ María Virginia Villegas Botero, ${ }^{3}$ \\ Carlos Gonzalo Robledo Restrepo y Aura Lucía Leal Castro ${ }^{5}$
}

Forma de citar

Villalobos Rodríguez AP, Díaz Ortega MH, Barrero Garzón LI, Rivera Vargas SM, Henríquez Iguarán DE, Villegas Botero MV, et al. Tendencias de los fenotipos de resistencia bacteriana en hospitales públicos y privados de alta complejidad de Colombia. Rev Panam Salud Publica. 2011;30(6):627-33.

RESUMEN Objetivo. Describir y comparar las frecuencias de los fenotipos de resistencia bacteriana de microorganismos obtenidos de pacientes en unidades de cuidados intensivos (UCI) y otros servicios de hospitalización (no UCI) públicos y privados de alta complejidad de Colombia.

Métodos. Estudio observacional, analítico, retrospectivo y multicéntrico, en el cual se consolidaron los registros de los aislamientos bacterianos y los fenotipos de resistencia bacteriana de los microorganismos obtenidos de pacientes atendidos en UCI y no UCI de 79 hospitales públicos y privados de alta complejidad en el periodo de enero de 2007 a diciembre de 2009. La información se analizó con el programa WHONET ${ }^{\circledR}$ versión 5.5 (OMS) de acuerdo con las recomendaciones del Instituto de Estándares Clínicos y de Laboratorio 2009 y se resumió en un formato de extracción de datos en Excel ${ }^{\circledR}$. Se realizó un análisis descriptivo en el cual se calcularon proporciones. El análisis de tendencias se realizó mediante la prueba de correlación de rangos de Spearman.

Resultados. Las tendencias de los fenotipos de resistencia bacteriana de 2007 a 2009 muestran un comportamiento incremental en la proporción de Enterococcus faecium resistente a vancomicina, Klebsiella pneumoniae resistente a imipenem y a ciprofloxacina, Escherichia coli resistente a ceftazidima, y Enterobacter cloacae resistente a cefotaxima $(\rho=1$, $\mathrm{P}<0,01)$ y una disminución de la proporción de los fenotipos E. coli resistente a ciprofloxacina, K. pneumoniae resistente a ceftazidima, Staphylococcus aureus resistente a oxacilina, y Pseudomonas aeruginosa resistente a ceftazidima y a ciprofloxacina $(\rho=-1, \mathrm{P}<0,01)$.

Conclusiones. El análisis de tendencias presentado en este estudio constituye la línea de base para el establecimiento de un subsistema nacional de vigilancia epidemiológica. Las tendencias observadas muestran que la resistencia bacteriana a los antimicrobianos en el ámbito hospitalario es un fenómeno dinámico en Colombia y son evidencia de la emergencia de los fenotipos Efa-van y Kpn-imp en los hospitales.

Palabras clave Agentes antibacterianos; farmacorresistencia bacteriana; vigilancia epidemiológica; hospitales; Colombia.

1 Instituto Nacional de Salud (INS), Subdirección de Vigilancia y Control en Salud Pública, Bogotá D.C., Colombia. La correspondencia se debe dirigir a Andrea Patricia Villalobos Rodríguez. Correo electrónico: avillalobos@ins.gov.co

2 Secretaría Distrital de Salud de Bogotá, Subsistema de Vigilancia Epidemiológica de Resistencia Bacteriana (SIVIBAC), Bogotá D.C., Colombia.
3 Centro Internacional de Entrenamiento e Investigaciones Médicas (CIDEIM), Santiago de Cali, Colombia.

4 Grupo para el Estudio de la Resistencia a Antibióticos de Medellín (GERMEN), Medellín, Colombia.

5 Universidad Nacional de Colombia, Grupo para el Control de la Resistencia Bacteriana en Bogotá (GREBO), Bogotá D.C., Colombia.
La resistencia bacteriana a los antimicrobianos en los servicios de salud se asocia a un aumento de la morbilidad, la mortalidad, la estancia hospitalaria y los costos de la atención sanitaria $(1,2)$. Por ello, se han tomado diversas medidas 
para su control, una de las cuales es generar información acerca del problema para cada una de las regiones y países (3, 4). Ya se ha documentado ampliamente el hallazgo de bacterias con resistencia a los antimicrobianos en centros hospitalarios $(5,6)$. La diseminación de estas bacterias constituye un punto crítico de la calidad de los servicios de salud y un reto importante para los profesionales y los entes reguladores involucrados en su contención.

La Estrategia Mundial de la OMS para la Contención de la Resistencia a los Antimicrobianos recomienda intervenciones para retrasar la aparición de resistencia a tales fármacos, así como reducir su diseminación (7); la vigilancia es el primer paso y la parte fundamental de esa estrategia (8). En Colombia, desde finales de la década de 2000, diversos grupos de investigación cuentan con experiencia en la caracterización de la resistencia bacteriana a los antimicrobianos en hospitales de las principales ciudades del país y en la divulgación de boletines epidemiológicos en el ámbito local (9). Sin embargo, no se disponía en la actualidad de datos consolidados a nivel nacional que describan la magnitud de la resistencia bacteriana en los hospitales.

En ese contexto, el presente estudio tiene como objetivo principal describir y comparar las frecuencias de los fenotipos de resistencia en bacterias grampositivas, enterobacterias y microorganismos gramnegativos no fermentadores obtenidos de pacientes en unidades de cuidados intensivos (UCI) y otros servicios de hospitalización diferentes a las UCI (no UCI) de hospitales públicos y privados de alta complejidad de Colombia, en el período de enero de 2007 a diciembre de 2009.

\section{MATERIALES Y MÉTODOS}

Se llevó a cabo un estudio observacional, analítico, retrospectivo y multicéntrico, en el cual se consolidaron los registros de los aislamientos bacterianos y los fenotipos de resistencia bacteriana de microorganismos grampositivos, enterobacterias y gramnegativos no fermentadores asociados a infección o colonización, obtenidos de muestras de sangre, orina, tracto respiratorio, tracto gastrointestinal, piel y tejidos blandos de pacientes hospitalizados en UCI y no UCI (excepto urgencias), de 79 hospitales de alta complejidad durante el período de enero de 2007 a diciembre de 2009. Del total de hospitales, 19 eran públicos y 60 privados, ubicados en 14 ciudades del territorio colombiano.

Las pruebas de identificación bioquímica y de susceptibilidad a los antimicrobianos (técnica de microdilución en caldo) fueron realizadas en los laboratorios de microbiología de las instituciones hospitalarias con los sistemas automatizados Dade Behring Microscan ${ }^{\circledR}$ y bioMérieux VITEK $^{\circledR}$. Todas las instituciones hospitalarias participantes realizaron control de calidad microbiológico interno y externo.

La información microbiológica generada en cada hospital se transfirió por medio del programa BackLink $2^{\circledR}$ (OMS) y fue consolidada por las siguientes entidades participantes: Subsistema de Vigilancia Epidemiológica de Resistencia Bacteriana de la Secretaría Distrital de Salud de Bogotá (SIVIBAC) $(n=49)$; Centro Internacional de Entrenamiento e Investigaciones Médicas (CIDEIM) $(n=10)$; Grupo para el Estudio de la Resistencia a Antibióticos de Medellín (GERMEN) ( $n=12)$, y Grupo para el Control de la Resistencia Bacteriana en Bogotá (GREBO) $(n=8)$. Para evitar datos duplicados en los casos en que los hospitales participaran en más de un grupo, al momento de generar los informes consolidados cada institución hospitalaria fue asignada sólo a una de las entidades participantes.

Las bases de datos se combinaron y analizaron con el programa $\mathrm{WHONET}^{\circledR}$ versión 5.5 (OMS) mediante la opción porcentaje de resistencia, teniendo en cuenta sólo el primer aislamiento por paciente. La clasificación de resistente se realizó de acuerdo a los puntos de corte definidos para cada combinación de microorganismo-antibiótico a partir de las recomendaciones del Instituto de Estándares Clínicos y de Laboratorio (CLSI, por su sigla en inglés) versión 2009.

El desenlace principal correspondió a los fenotipos de resistencia bacteriana seleccionados mediante un consenso de expertos, incluidos los principales agentes bacterianos causantes de infecciones asociadas a la atención sanitaria en el ámbito hospitalario, entendiéndose como fenotipo de resistencia la combinación de un agente bacteriano (género y especie) y la resistencia a un determinado antibiótico.

Cada entidad participante documentó el consolidado de los desenlaces de interés del conjunto de hospitales adscritos a su grupo en un formato de extracción de datos diseñado en Excel ${ }^{\circledR}$. La información documentada en los formatos de extracción de datos se consolidó en una base de datos en Excel ${ }^{\circledR}$.

Se realizó un análisis descriptivo mediante el cual se calcularon las proporciones anuales de los aislamientos bacterianos y los fenotipos de resistencia bacteriana con un nivel de confianza de $95 \%$. Para el contraste de hipótesis se empleó la prueba de ji al cuadrado $\left(\chi^{2}\right)$ de comparación de proporciones en muestras independientes. El análisis de tendencias se realizó mediante la prueba de correlación de rangos de Spearman con un nivel de significación estadística de 0,01 . Todos los análisis se llevaron a cabo con el programa StataSE ${ }^{\circledR} 10$.

\section{Consideraciones éticas}

Esta investigación se rigió de acuerdo a las normas científicas, técnicas y administrativas para la investigación en salud del Ministerio de Salud de la República de Colombia, Resolución No. 8430 del 4 de Octubre de 1993. El estudio se clasificó como una investigación sin riesgo y no requirió consentimiento informado. El manejo de los datos mantuvo estrictos parámetros de confidencialidad de acuerdo a las leyes vigentes.

\section{RESULTADOS}

Se consolidaron 233120 registros de aislamientos bacterianos y 415551 registros de fenotipos de resistencia bacteriana.

El análisis de la distribución del total de aislamientos bacterianos en los servicios no UCI muestra que los de Escherichia coli se aislaron con mayor frecuencia en los tres años de estudio, seguido de los aislamientos de Staphyococcus aureus (cuadro 1). En las UCI, el microorganismo más frecuente en los tres años de estudio correspondió igualmente a cepas de E. coli. Para 2007, los aislados de S. aureus estuvieron en segundo lugar en orden de frecuencia, mientras que para 2008 y 2009, fueron cepas de Klebsiella pneumoniae (véase el cuadro 1).

Se observó que los aislamientos de Staphylococcus epidermidis resistente a oxacilina (Sep-oxa), S. aureus resistente a oxacilina (Sau-oxa), Enterobacter cloacae resistente a cefotaxima (Eclo-ctx) y Acinetobacter baumannii resistente a imipenem (Aba-imp) fueron los fenotipos de mayor frecuencia en los servicios no UCI para los tres años de estudio (cuadro 2). 
CUADRO 1. Número y porcentaje de aislamientos bacterianos obtenidos de pacientes en servicios no UCl y en las UCl de hospitales públicos y privados de alta complejidad, Colombia, por año, 2007 a 2009

\begin{tabular}{|c|c|c|c|c|c|c|c|c|c|c|}
\hline \multirow[b]{2}{*}{ Servicío } & \multirow[b]{2}{*}{ Microorganismo } & \multicolumn{3}{|c|}{2007} & \multicolumn{3}{|c|}{2008} & \multicolumn{3}{|c|}{2009} \\
\hline & & No. & $\%$ & IC 95\% & No. & $\%$ & IC 95\% & No. & $\%$ & IC 95\% \\
\hline \multirow[t]{10}{*}{ No UCl } & Staphylococcus aureus & 6886 & 12,9 & $12,6-13,2$ & 6820 & 13,5 & $13,2-13,8$ & 6451 & 12,8 & $12,5-13,1$ \\
\hline & Enterococcus faecalis & 2006 & 3,8 & $3,6-3,9$ & 2228 & 4,4 & $4,2-4,6$ & 2393 & 4,7 & $4,6-4,9$ \\
\hline & Enterococcus faecium & 322 & 0,6 & $0,5-0,7$ & 311 & 0,6 & $0,5-0,7$ & 390 & 0,8 & $0,7-0,8$ \\
\hline & Escherichia coli & 18419 & 34,6 & $34,2-35,0$ & 19963 & 39,6 & $39,2-40,0$ & 19694 & 39,0 & $38,6-39,4$ \\
\hline & Klebsiella pneumoniae & 4445 & 8,4 & $8,1-8,6$ & 4923 & 9,8 & $9,5-10,0$ & 4992 & 9,9 & $9,6-10,1$ \\
\hline & Enterobacter aerogenes & 523 & 1,0 & $0,9-1,1$ & 539 & 1,1 & $1,0-1,2$ & 531 & 1,1 & $1,0-1,1$ \\
\hline & Acinetobacter baumannii & 876 & 1,7 & $1,5-1,8$ & 635 & 1,3 & $1,2-1,4$ & 712 & 1,4 & $1,3-1,5$ \\
\hline & Pseudomonas aeruginosa & 3129 & 5,9 & $5,7-6,1$ & 3436 & 6,8 & $6,6-7,0$ & 3332 & 6,6 & $6,4-6,8$ \\
\hline & Otros & 9421 & 17,7 & $17,4-18,0$ & 3528 & 7,0 & $6,8-7,2$ & 4346 & 8,6 & $8,4-8,8$ \\
\hline & Total & 53249 & 100 & - & 50392 & 100 & - & 50515 & 100 & - \\
\hline \multirow{9}{*}{$\mathrm{UCl}$} & Escherichia coli & 4568 & 17,6 & $17,2-18,1$ & 4601 & 17,3 & $16,9-17,8$ & 4929 & 18,6 & $18,1-19,1$ \\
\hline & Klebsiella pneumoniae & 3461 & 13,4 & $12,9-13,8$ & 3575 & 13,5 & $13,1-13,9$ & 3661 & 13,8 & $13,4-14,2$ \\
\hline & Proteus mirabilis & 691 & 2,7 & $2,5-2,9$ & 718 & 2,7 & $2,5-2,9$ & 787 & 3,0 & $2,8-3,2$ \\
\hline & Enterobacter cloacae & 1280 & 4,9 & $4,7-5,2$ & 1249 & 4,7 & $4,5-5,0$ & 1177 & 4,4 & $4,2-4,7$ \\
\hline & Enterobacter aerogenes & 342 & 1,3 & $1,2-1,5$ & 374 & 1,4 & $1,3-1,6$ & 330 & 1,3 & $1,1-1,4$ \\
\hline & Acinetobacter baumannii & 1061 & 4,1 & $3,9-4,3$ & 848 & 3,2 & $3,0-3,4$ & 824 & 3,1 & $2,9-3,3$ \\
\hline & Pseudomonas aeruginosa & 2247 & 8,7 & $8,3-9,0$ & 2247 & 8,5 & $8,1-8,8$ & 2284 & 8,6 & $8,3-9,0$ \\
\hline & Otros & 4017 & 15,5 & $15,1-15,9$ & 5727 & 21,6 & $21,1-22,1$ & 5501 & 20,8 & $20,3-21,2$ \\
\hline & Total & 25915 & 100 & - & 26537 & 100 & - & 26512 & 100 & - \\
\hline
\end{tabular}

UCl: unidades de cuidados intensivos.

CUADRO 2. Número y porcentaje de los fenotipos de resistencia bacteriana en microorganismos grampositivos, enterobacterias y gramnegativos no fermentadores obtenidos de pacientes en servicios no UCI de hospitales públicos y privados de alta complejidad, Colombia, por año, 2007 a 2009

\begin{tabular}{|c|c|c|c|c|c|c|c|c|c|c|}
\hline \multirow[b]{2}{*}{ Microorganismo } & \multirow{2}{*}{$\begin{array}{c}\text { Fenotipo } \\
\text { de resistencia }\end{array}$} & \multicolumn{3}{|c|}{2007} & \multicolumn{3}{|c|}{2008} & \multicolumn{3}{|c|}{2009} \\
\hline & & No. & $\%$ & IC $95 \%$ & No. & $\%$ & IC 95\% & No. & $\%$ & IC 95\% \\
\hline \multirow[t]{2}{*}{ Gram positivos } & Sau-oxa & 6521 & 38,2 & $37,0-39,3$ & 6463 & 36,4 & $35,2-37,6$ & 3572 & 36,8 & $35,2-38,4$ \\
\hline & Sep-oxa & 3064 & 73,8 & $72,2-75,3$ & 3575 & 75,7 & $74,3-77,1$ & 2134 & 76,2 & $74,4-78,0$ \\
\hline \multirow[t]{9}{*}{ Enterobacterias } & Eco-caz & 15100 & 6,1 & $5,8-6,5$ & 17824 & 6,3 & $6,0-6,7$ & 23296 & 6,7 & $6,4-7,1$ \\
\hline & Eco-ctx & 12804 & 6,3 & $5,9-6,8$ & 13707 & 6,9 & $6,5-7,4$ & 17395 & 7,0 & $6,6-7,4$ \\
\hline & Kpn-ctx & 3250 & 28,5 & $26,9-30,1$ & 3307 & 29,1 & $27,5-30,6$ & 2447 & 26,6 & $24,9-28,4$ \\
\hline & Kpn-imp & 4044 & 0,6 & $0,4-0,9$ & 4612 & 2,3 & $1,8-2,7$ & 4445 & 2,3 & $1,8-2,7$ \\
\hline & Kpn-cip & 4036 & 12,6 & $11,5-13,6$ & 4664 & 13,9 & $12,8-14,9$ & 4657 & 15,4 & $14,3-16,4$ \\
\hline & Eclo-caz & 1478 & 32,4 & $30,0-34,8$ & 2077 & 25,4 & $23,5-27,3$ & 1266 & 29,5 & $26,9-32,0$ \\
\hline & Eclo-ctx & 1267 & 38,4 & $35,6-41,1$ & 1316 & 38,9 & $36,2-41,6$ & 793 & 44,8 & $41,2-48,3$ \\
\hline & Eclo-imp & 1707 & 1,0 & $0,5-1,5$ & 1595 & 1,8 & $1,1-2,5$ & 1336 & 1,1 & $0,5-1,7$ \\
\hline & Eclo-cip & 1696 & 28,0 & $25,8-30,1$ & 1921 & 23,7 & $21,8-25,7$ & 1373 & 28,8 & $26,4-31,3$ \\
\hline $\begin{array}{l}\text { Gram negativos } \\
\text { no fermentadores }\end{array}$ & Aba-imp & 754 & 47,9 & $44,2-51,5$ & 543 & 47,2 & $42,9-51,4$ & 404 & 45,5 & $40,6-50,5$ \\
\hline
\end{tabular}

UCl: unidades de cuidados intensivos.

Sau-oxa: Staphylococcus aureus resistente a oxacilina.

Sep-oxa: Staphylococcus epidermidis resistente a oxacilina.

Efa-van: Enterococcus faecium resistente a vancomicina.

Eco-caz: Escherichia coli resistente a ceftazidima.

Eco-ctx: E. coli resistente a cefotaxima.

Eco-cip: E. coli resistente a ciprofloxacina.
Kpn-caz: Klebsiella pneumoniae resistente a ceftazidima

Kpn-ctx: K. pneumoniae resistente a cefotaxima.

Kpn-imp: K. pneumoniae resistente a imipenem.

Kpn-cip: K. pneumoniae resistente a ciprofloxacina.

Eclo-caz: Enterobacter cloacae resistente a ceftazidima.

Eclo-ctx: E. cloacae resistente a cefotaxima.

Eclo-imp: E. cloacae resistente a imipenem.
Eclo-cip: E. cloacae resistente a ciprofloxacina.

Psa-caz: Pseudomonas aeruginosa resiente a ceftazidima. Psa-imp: P. aeruginosa resistente a imipenem.

Psa-cip: P. aeruginosa resistente a ciprofloxacina

Aba-imp: Acinetobacter baumannii resistente a imipenem. 
En las UCI, los fenotipos obtenidos con mayor frecuencia en los tres años de estudio fueron Sep-oxa, Eclo-ctx y Abaimp (cuadro 3).

En los servicios no UCI se presentaron diferencias estadísticamente significativas $(P<0,01)$ en las proporciones para los tres años de estudio de los fenotipos Enterococcus faecium resistente a vancomicina (Efa-van); E. coli resistente a ciprofloxacina (Eco-cip); K. pneumoniae resistente a ceftazidima (Kpn-caz), imipenem (Kpn-imp) y ciprofloxacina (Kpncip), E. cloacae resistente a ceftazidima (Eclo-caz), cefotaxima (Eclo-ctx) y ciprofloxacina (Eclo-cip) y Pseudomonas aeruginosa resistente a ciprofloxacina (Psa-cip) (véase el cuadro 2). Un análisis posterior permitió determinar que la proporción del fenotipo Efa-van aumentó en 152,5\% entre 2007 y 2008, y en 154,4\% entre 2008 y 2009. Asimismo, se observó que la proporción del fenotipo Kpn-imp aumentó 283,3\% entre 2007 y 2008.

En las UCI se hallaron diferencias estadísticamente significativas $(P<0,01)$ en las proporciones para los tres años de estudio de los fenotipos Sau-oxa, Efa-van, E. coli resistente a ceftazidima (Eco-caz), Eco-cip, Kpn-imp, Kpn-cip, $P$. aeruginosa resistente a ceftazidima (Psa- caz) y Psa-cip (véase el cuadro 3). Un análisis posterior permitió establecer que la proporción de Efa-van en las UCI aumentó en $64,7 \%$ entre 2007 y 2008, y en $55,9 \%$ entre 2008 y 2009. Por su parte la proporción de Kpn-imp se incrementó en $130,8 \%$ entre 2007 y 2008.

En los tres años estudiados, la frecuencia de los fenotipos de resistencia bacteriana en los servicios no UCI mostraron tendencia al aumento de Efa-van, Kpn-imp, Kpn-cip y Eclo-ctx $(\rho=1$, $P<0,01)$, así como un patrón decreciente en la proporción de Eco-cip y Kpn-caz $\rho=-1, P<0,01$ ) (figura 1). En cuanto a las frecuencias de los fenotipos de resistencia bacteriana en las UCI, en el mismo período de estudio se observó un aumento en la proporción de Efavan, Eco-caz, Kpn-imp, Kpn-cip ( $\rho=1$, $P<0,01)$ y una reducción en la proporción de los fenotipos Sau-oxa, Psa-caz y Psa-cip ( $\rho=-1, P<0,01)$ (figura 2 ).

\section{DISCUSIÓN}

Este estudio permitió mostrar un incremento simultáneo en la proporción de aislamientos de $K$. pneumoniae resistente a cefotaxima y $K$. pneumoniae resistente a ciprofloxacina en las UCI. Como se ha confirmado en estudios previos $(10,11)$ es posible que estos eventos correspondan a la circulación de cepas con corresistencia mediada por la expresión de betalactamasas de espectro extendido (BLEE) y mecanismos de resistencia a quinolonas (mutaciones en los genes que codifican para DNA girasa y topoisomerasa IV, alteraciones en la membrana externa bacteriana y sistemas de expulsión de antibióticos). Ante el riesgo de fracaso terapéutico que conlleva este fenómeno, es importante considerar la necesidad de vigilar la resistencia de esta especie a múltiples antibióticos.

La utilización frecuente de fármacos carbapenémicos en los servicios de salud de Colombia ha tenido un impacto en la aparición de la resistencia a estos antibióticos de última línea, especialmente entre cepas de K. pneumoniae cuya frecuencia de resistencia a imipenem representó en este estudio variaciones porcentuales superiores al $100 \%$. Estos hallazgos se pueden relacionar con la ocurrencia de un brote por cepas de K. pneumoniae productora de carbapenemasa KPC-3 caracterizado a mediados de 2008, que afectó a 84 pacientes en un hospital de tercer nivel en la ciudad de Medellín, institución en la cual se originó el primer informe sobre

CUADRO 3. Número y porcentaje de los fenotipos de resistencia bacteriana en microorganismos grampositivos, enterobacterias y gramnegativos no fermentadores obtenidos de pacientes en las UCI de hospitales públicos y privados de alta complejidad, Colombia, por año, 2007 a 2009

\begin{tabular}{|c|c|c|c|c|c|c|c|c|c|c|}
\hline & \multirow{2}{*}{$\begin{array}{c}\text { Fenotipo } \\
\text { de resistencia }\end{array}$} & \multicolumn{3}{|c|}{2007} & \multicolumn{3}{|c|}{2008} & \multicolumn{3}{|c|}{2009} \\
\hline & & No. & $\%$ & IC 95\% & No. & $\%$ & IC 95\% & No. & $\%$ & IC 95\% \\
\hline \multirow[t]{3}{*}{ Gram positivos } & Sau-oxa & 3432 & 40,0 & $38,4-41,7$ & 3232 & 37,1 & $35,4-38,8$ & 2918 & 28,1 & $26,5-29,7$ \\
\hline & Sep-oxa & 2911 & 81,3 & $79,8-82,7$ & 3000 & 80,8 & $79,4-82,3$ & 2693 & 79,3 & $77,7-80,8$ \\
\hline & Efa-van & 137 & 10,2 & $4,8-15,6$ & 190 & 16,8 & $11,3-22,4$ & 195 & 26,2 & $19,7-32,6$ \\
\hline \multirow[t]{11}{*}{ Enterobacterias } & Eco-caz & 3631 & 8,2 & $7,3-9,1$ & 3869 & 9,4 & $8,5-10,3$ & 4549 & 10,2 & $9,3-11,1$ \\
\hline & Eco-ctx & 3269 & 9,2 & $8,2-10,2$ & 3356 & 10,1 & $9,0-11,1$ & 3571 & 9,9 & $8,9-11,0$ \\
\hline & Eco-cip & 3951 & 28,2 & $26,8-29,6$ & 4438 & 24,9 & $23,6-26,1$ & 4734 & 25,7 & $24,5-27,0$ \\
\hline & Kpn-caz & 2856 & 28,1 & $26,5-29,8$ & 3399 & 26,8 & $25,3-28,3$ & 3445 & 28,2 & $26,6-29,7$ \\
\hline & Kpn-ctx & 2597 & 28,3 & $26,6-30,1$ & 2797 & 29,1 & $27,4-30,7$ & 2507 & 31,3 & $29,4-33,1$ \\
\hline & Kpn-imp & 3012 & 1,3 & $0,8-1,7$ & 3532 & 3,0 & $2,5-3,6$ & 3542 & 4,0 & $3,4-4,7$ \\
\hline & Kpn-cip & 2899 & 10,2 & $9,1-11,4$ & 3519 & 12,9 & $11,8-14,0$ & 3524 & 14,3 & $13,1-15,5$ \\
\hline & Eclo-caz & 1009 & 30,5 & $27,6-33,4$ & 1111 & 25,7 & $23,1-28,4$ & 980 & 27,9 & $25,0-30,7$ \\
\hline & Eclo-ctx & 898 & 39,1 & $35,8-42,3$ & 839 & 36,8 & $33,5-40,2$ & 709 & 40,3 & $36,6-44,0$ \\
\hline & Eclo-imp & 1201 & 2,1 & $1,2-2,9$ & 1273 & 1,9 & $1,1-2,7$ & 1133 & 1,4 & $0,7-2,1$ \\
\hline & Eclo-cip & 1192 & 18,2 & $16,0-20,4$ & 1277 & 14,3 & $12,4-16,3$ & 1154 & 16,8 & $14,6-19,0$ \\
\hline \multirow{4}{*}{$\begin{array}{l}\text { Gram negativos } \\
\text { no fermentadores }\end{array}$} & Psa-caz & 1967 & 31,2 & $29,1-33,2$ & 2150 & 26,2 & $24,3-28,1$ & 2095 & 23,6 & $21,8-25,5$ \\
\hline & Psa-imp & 2040 & 25,9 & $24,0-27,8$ & 2258 & 25,6 & $23,7-27,4$ & 2219 & 24,0 & $22,2-25,8$ \\
\hline & Psa-cip & 2039 & 28,5 & $26,6-30,5$ & 2245 & 25,6 & $23,8-27,4$ & 2222 & 23,7 & $21,9-25,5$ \\
\hline & Aba-imp & 1002 & 56,5 & $53,4-59,6$ & 831 & 58,5 & $55,7-61,9$ & 764 & 62,6 & $59,1-66,1$ \\
\hline
\end{tabular}

UCl: unidades de cuidados intensivos.

Sau-oxa: Staphylococcus aureus resistente a oxacilina.

Sep-oxa: Staphylococcus epidermidis resistente a oxacilina.

Efa-van: Enterococcus faecium resistente a vancomicina.

Eco-caz: Escherichia coli resistente a ceftazidima.

Eco-ctx: E. coli resistente a cefotaxima.

Eco-cip: E. coli resistente a ciprofloxacina.
Kpn-caz: Klebsiella pneumoniae resistente a ceftazidima. Kpn-ctx: K. pneumoniae resistente a cefotaxima. Kpn-imp: K. pneumoniae resistente a imipenem. Kpn-cip: K. pneumoniae resistente a ciprofloxacina. Eclo-caz: Enterobacter cloacae resistente a ceftazidima. Eclo-ctx: E. cloacae resistente a cefotaxima. Eclo-imp: E. cloacae resistente a imipenem.
Eclo-cip: E. cloacae resistente a ciprofloxacina.

Psa-caz: Pseudomonas aeruginosa resiente a ceftazidima. Psa-imp: $P$. aeruginosa resistente a imipenem. Psa-cip: $P$. aeruginosa resistente a ciprofloxacina. Aba-imp: Acinetobacter baumannii resistente a imipenem. 
FIGURA 1. Porcentaje de resistencia bacteriana en microorganismos obtenidos de pacientes en servicios no UCl de hospitales públicos y privados de alta complejidad, según fenotipo, Colombia, por año, 2007 a 2009

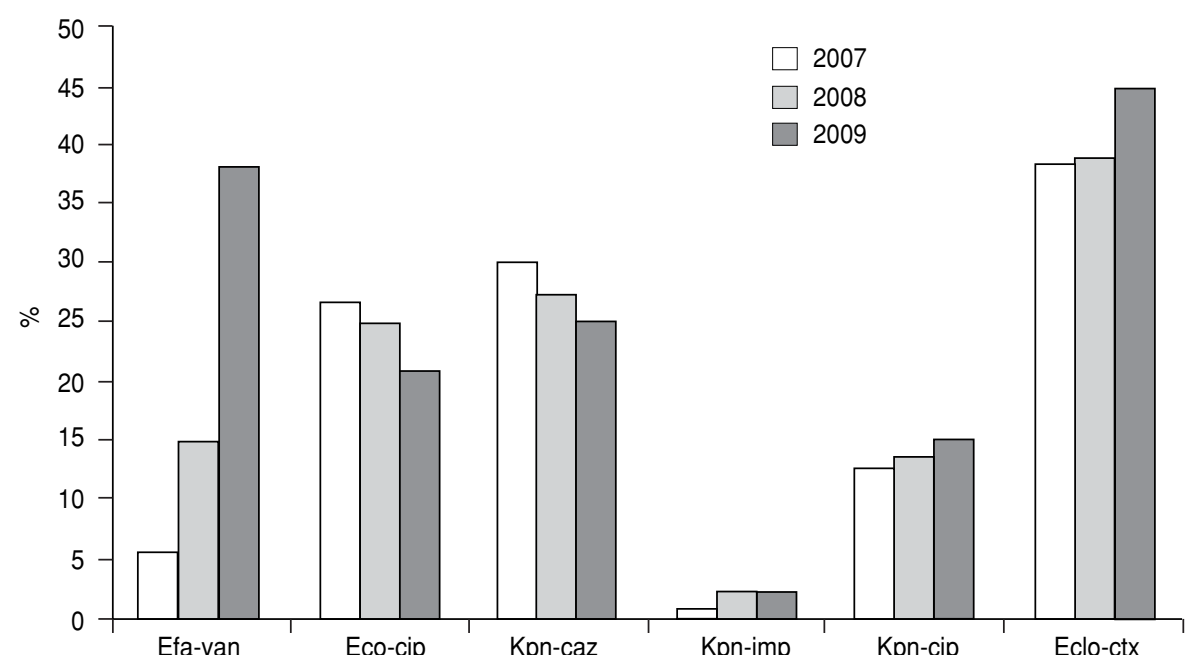

UCl: unidades de cuidados intensivos.

$P<0,01$.

Efa-van: Enterococcus faecium resistente a vancomicina.

Eco-cip: Escherichia coli resistente a ciprofloxacina.

Kpn-caz: Klebsiella pneumoniae resistente a ceftazidima.

Kpn-imp: K. pneumoniae resistente a imipenem.

Kpn-cip: K. pneumoniae resistente a ciprofloxacina.

Eclo-ctx: Enterobacter cloacae resistente a cefotaxima.

FIGURA 2. Porcentaje de los fenotipos de resistencia bacteriana en microorganismos obtenidos de pacientes en las UCl de hospitales públicos y privados de alta complejidad, Colombia, por año, 2007 a 2009

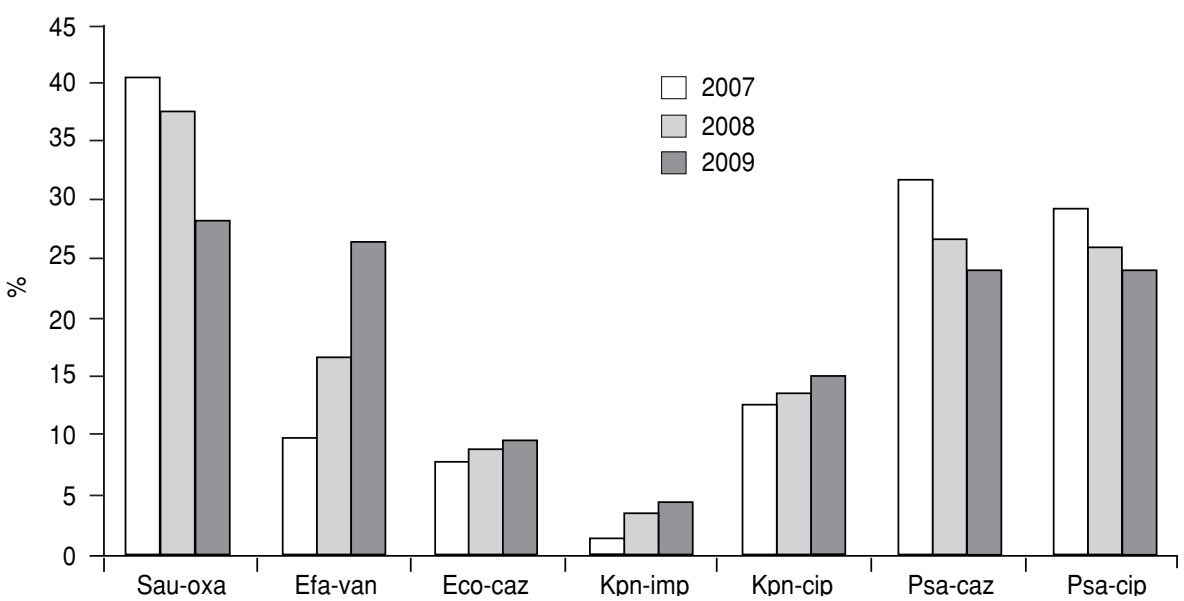

UCl: unidades de cuidados intensivos.

$$
P<0,01 \text {. }
$$

Sau-oxa: Staphylococcus aureus resistente a oxacilina.

Efa-van: Enterococcus faecium resistente a vancomicina.

Eco-caz: Escherichia coli resistente a ceftazidima.

Kpn-imp: K. pneumoniae resistente a imipenem.

Kpn-cip: K. pneumoniae resistente a ciprofloxacina.

Psa-caz: Pseudomonas aeruginosa resiente a ceftazidima.

Psa-cip: P. aeruginosa resistente a ciprofloxacina.

la presencia de cepas de K. pneumoniae productora de KPC-2 en Latinoamérica (12). No obstante, a pesar de la aparición de aislamientos de K. pneumoniae productores de KPC en Colombia, el incremento de la resistencia a imipenem observada en el presente estudio podría ser resultado de la aparición de aislamientos con expresión de otros mecanismos de resistencia, como alteraciones de porina, los cuales han sido documentados en otros estudios (13).
Por otra parte, la especie E. cloacae poco a poco ha cobrado importancia en la epidemiología hospitalaria, por ser una fuente importante de resistencia transferible (14). El presente estudio muestra un aumento en la frecuencia de aislamientos de E. cloacae resistente a cefotaxima en servicios no UCI, así como una proporción importante del fenotipo en las UCI. Estos resultados, que están en el rango de lo documentado por GREBO en hospitales de Bogotá (30\% a $50 \%$ ) (15), permiten sugerir un posible aumento de la presentación de aislamientos con hiperexpresión de betalactamasa cromosómica inducible AmpC o producción de BLEE. Es necesario prevenir la diseminación de las cepas de E. cloacae resistente a los antibióticos, especialmente a cefalosporinas de tercera generación, evento que puede pasar inadvertido y convertirse en un fenómeno difícil de contener en el medio hospitalario.

Con respecto a la especie E. faecium, se observó un incremento significativo en la frecuencia de la resistencia a vancomicina, tanto en los servicios no UCI como en las UCI, con variaciones porcentuales superiores a $100 \%$. La aparición rápida de esta resistencia se ha documentado en otros países, como lo muestra el estudio de Chong en 2000 en Corea (16), en el que la frecuencia pasó de menos de $5 \%$ en 1996 a casi $30 \%$ en 1999 . Aunque se cuenta con datos que muestran un aumento importante en la frecuencia de cepas de E. faecium resistente a vancomicina, se debe considerar que no existe evidencia nacional publicada acerca de la ocurrencia de brotes por ese microorganismo en el ámbito hospitalario para el período 2007 a 2009. Además, la circulación de ese fenotipo no está generalizada en el país, según señalan los datos publicados en el boletín epidemiológico de SIVIBAC 2008-2009, pero está presente en $25 \%$ de las instituciones hospitalarias de alta complejidad. No obstante, es importante tener en cuenta que este microorganismo puede constituirse en un problema de salud pública por lo difícil que es tratar las infecciones que causa, y por la posibilidad de transferencia de esta resistencia a otros gérmenes de importancia nosocomial, como son las cepas de S. aureus.

Se observó una disminución del porcentaje de aislamientos de $S$. aureus resistente a oxacilina en las UCI; este comportamiento es coherente con lo presentado por SIVIBAC (17) y lo que figura en el in- 
forme de vigilancia del ECDC (6). Aunque es probable que las medidas para prevenir la transmisión de este fenotipo en las unidades de cuidados intensivos hayan logrado disminuir su circulación, no se cuenta con evidencia nacional que así lo demuestre.

Este estudio consolidó datos de iniciativas de vigilancia de la resistencia a los antibióticos, e información sobre un número importante de aislamientos, lo cual determina una ventaja para describir las tendencias de los porcentajes de resistencia y una mayor precisión de las estimaciones. No obstante, cuando se realizan estudios que involucran instituciones hospitalarias con características heterogéneas, la frecuencia de algunos fenotipos de resistencia bacteriana podría pasar inadvertida, por lo cual es imprescindible conocer la epidemiología institucional.

Las tendencias ascendentes de las frecuencias de los fenotipos de resistencia bacteriana identificados en el presente estudio son consecuencia de diversos factores, cuyo análisis está fuera del objetivo planteado; entre ellos, el uso excesivo e inadecuado de antibióticos quizás sea el factor más importante en el desarrollo de resistencia a esos medicamentos (18). Como fenómeno ecológico, la resistencia bacteriana a los antimicrobianos debe correlacionarse con las intervenciones locales $(19,20)$, particularmente, el análisis de la tendencia de la resistencia, que es más fácil de comprender si se evalúan los datos en el contexto del uso local de los antimicrobianos. Desafortunadamente hay muy pocos estudios a nivel nacional sobre el consumo de antibióticos en la mayoría de instituciones hospitalarias.

Se debe considerar que los resultados de las pruebas de susceptibilidad a los antimicrobianos que integran este estudio se obtuvieron de agentes colonizadores e infecciosos. A pesar de que esta información debe ser interpretada con precaución como insumo para la aplicación de pautas terapéuticas, es muy útil para tomar medidas de control, ya que refleja de manera más representativa la ecología bacteriana del ámbito hospitalario.

\section{Conclusiones}

Esta es la primera publicación de datos consolidados a nivel nacional respecto a la situación de la resistencia bacteriana a los antimicrobianos en el ámbito hospitalario. El análisis de la magnitud y las tendencias de la resistencia bacteriana a los antimicrobianos presentado en este estudio constituye la línea de base para la instauración de un subsistema nacional de vigilancia epidemiológica y el diseño de políticas, programas y acciones orientadas a su prevención y control.

El presente estudio detectó un aumento en la frecuencia de los fenotipos Efa-van, Kpn-imp, Kpn-cip, Eco-caz y Eclo-ctx, así como una disminución de la frecuencia de los fenotipos Eco-cip, Kpn-caz, Sau-oxa, Psa-caz y Psa-cip. Estas tendencias señalan que la resistencia bacteriana a los antimicrobianos en el ámbito hospitalario es un fenómeno dinámico en Colombia y son evidencia de la emergencia de los fenotipos Efa-van y Kpn-imp en los hospitales.
La contención de la diseminación de estos microorganismos en los hospitales debe convertirse en una prioridad institucional. Con base en la evidencia, se recomienda: tomar precauciones de contacto de pacientes colonizados o infectados; aplicar medidas de control para prevenir la transmisión de infecciones de persona a persona, como son el aislamiento del paciente, uso de elementos protectores (guantes, bata, otros) e higiene adecuada de las manos; desarrollo de guías y programas de uso prudente de antibióticos; educación del personal de salud, y otras intervenciones de acuerdo a la epidemiología institucional, como la vigilancia activa de portadores. Para los laboratorios de microbiología se recomienda implementar normas del CLSI y contar con métodos de desempeño operativo adecuados para la identificación bioquímica y evaluación de la susceptibilidad a los antimicrobianos, así como la participación en programas de control de calidad y la confirmación molecular de los fenotipos de resistencia bacteriana.

Agradecimientos. Los autores expresan su gratitud a cada una de las personas involucradas en las fases de la realización de este estudio, tanto en el Ministerio de la Protección Social e Instituto Nacional de Salud, como en cada una de las instituciones hospitalarias. Este trabajo fue financiado con recursos del Convenio Interadministrativo 081 de 2010 realizado entre el Ministerio de la Protección Social y el Instituto Nacional de Salud.

\section{REFERENCIAS}

1. Cosgrove SE. The relationship between antimicrobial resistance and patient outcomes: mortality, length of hospital stay, and health care costs. Clin Infect Dis. 2006;15;42(Suppl 2):S82-9.

2. Wilson SJ, Knipe CJ, Zieger MJ, Gabehart KM, Goodman JE, Volk HM, et al. Direct costs of multidrug-resistant Acinetobacter baumannii in the burn unit of a public teaching hospital. Am J Infect Control. 2004;32(6):342-4.

3. Tenover FC. Mechanisms of antimicrobial resistance in bacteria. Am J Infect Control. 2006;34(5 Suppl 1):S3-10; discussion S64-73.

4. Kaye KS, Engemann JJ, Fraimow HS, Abrutyn E. Pathogens resistant to antimicrobial agents: epidemiology, molecular mechanisms, and clinical management. Infect Dis Clin North Am. 2004;18(3):467-511.

5. World Health Organization. World Health Organization: Fifty-first World Health Assembly item 21.3, Emerging and other communicable diseases: Antimicrobial resistance. Ginebra: OMS; 1998. (WHA51.17, agenda item 21.3).

6. European Centre for disease prevention and control (ECDC). Annual epidemiological report on communicable diseases in Europe. Estocolmo: EDCD; 2010. (Surveillance report: Annual Epidemiological report on communicable diseases in Europe).

7. Organización Mundial de la Salud. Estrategia mundial de la OMS para contener la resis- tencia a los antimicrobianos. WHO/CDS/ CSR/2001.2.

8. McGowan JE. Antibiotic resistance in hospital bacteria: current patterns, modes of appearance or spread and economic impact. Rev Med Microbiol. 1999;2:161-9.

9. Ministerio de la Protección Social. Caracterización de entidades, redes y grupos de investigación en infecciones intrahospitalarias y resistencia bacteriana. [en línea]. Bogotá D.C.: Ministerio de la Protección Social; 2009. Disponible en: http://www.ins.gov. co/?idcategoria $=88860$ Acceso el 27 de enero de 2011.

10. Paterson DL, Mulazimoglu L, Casellas JM, Ko WC, Goossens H, Von Gottberg A, et al. 
Epidemiology of ciprofloxacin resistance and its relationship to extended-spectrum betalactamase production in Klebsiella pneumoniae isolates causing bacteremia. Clin Infect Dis. 2000;30(3):473-8.

11. Villegas MV, Correa A, Perez F, Zuluaga T, Radice M, Gutkind G, et al. CTX-M-12 beta-lactamase in a Klebsiella pneumoniae clinical isolate in Colombia. Antimicrob Agents Chemother. 2004;48(2):629-31.

12. Villegas MV, Lolans K, Correa A, Suarez CJ, Lopez JA, Vallejo M, et al. First detection of the plasmid-mediated class A carbapenemase KPC-2 in clinical isolates of Klebsiella pneumoniae from South America. Antimicrob Agents Chemother. 2006;50(8):2880-2.

13. Ardanuy $C$, Linares J, Dominguez MA, Hernandez-Alles S, Benedi VJ, MartinezMartinez L. Outer membrane profiles of clonally related Klebsiella pneumoniae isolates from clinical samples and activities of cephalosporins and carbapenems. Antimicrob Agents Chemother. 1998;42(7):1636-40.

14. Sanders WE Jr, Sanders CC. Enterobacter spp.: pathogens poised to flourish at the turn of the century. Clin Microbiol Rev. 1997;10(2):220-41.

15. GREBO - Grupo de Estudio de la Resistencia en Bogotá [internet]. Bogotá D.C.: grebo.org; 2011. Disponible en: http://www.grebo.org/ Acceso el 2 de marzo de 2011.

16. Chong Y, Lee K. Present situation of antimicrobial resistance in Korea. J Infect Chemother. 2000;6(4):189-95.

17. Secretaría Distrital de Salud de Bogotá. Boletín Epidemiológico de Resistencia Bacteriana (SIVIBAC) 2008-2009. [en línea]. Bogotá D.C.: Secretaria Distrital de Salud de Bogotá; 2008. Disponible en: http:/ / www.saludcapital gov.co/sitios/VigilanciaSaludPublica/Site
CollectionDocuments/Bolet $\%$ C $3 \%$ ADn $\% 20$ SIVIBAC\%202008\%20-\%202009.pdf Acceso el 28 de enero de 2011.

18. McCaig LF, Hughes JM. Trends in antimicrobial drug prescribing among office-based physicians in the United States. JAMA. 1995;18;273(3):214-9.

19. Serefhanoglu K, Turan H, Timurkaynak FE, Arslan $\mathrm{H}$. Bloodstream infections caused by ESBL-producing E. coli and K. pneumoniae: risk factors for multidrug-resistance. Braz J Infect Dis. 2009;13(6):403-7.

20. Slama TG. Gram-negative antibiotic resistance: there is a price to pay. Crit Care. 2008; 12(Suppl 4):S1-7.

Manuscrito recibido el 1 de mayo de 2011. Aceptado para publicación, tras revisión, el 28 de septiembre de 2011.

ABSTRACT Objective. Describe and compare the frequency of bacterial resistance phenotypes of microorganisms obtained from patients in intensive care units (ICU) and other (nonICU) high-complexity public and private hospital services in Colombia.

Trends of bacterial resistance Methods. A retrospective observational, analytical, multicenter study was conducted. The records from January 2007 to December 2009 on bacterial isolates and bacterial resistance phenotypes of microorganisms obtained from ICU and nonICU patients in 79 high-complexity public and private hospitals were consolidated. The information was analyzed with the WHONET ${ }^{\circledR} 5.5$ (WHO) software, following the 2009 recommendations of the Clinical and Laboratory Standards Institute, and summarized on an Excel $^{\circledR}$ spreadsheet. A descriptive analysis with the calculation of proportions was performed. The trends were analyzed with Spearman rank correlation.

Results. The 2007-2009 trends for bacterial resistance phenotypes show increased percentages of vancomycin-resistant Enterococcus faecium, imipenem-resistant Klebsiella pneumoniae, ciprofloxacin-resistant K. pneumoniae, ceftazidime-resistant Escherichia coli and cefotaxime-resistant Enterobacter cloacae $(\rho=1, P<0.01)$, and reduced percentages of ciprofloxacin-resistant $E$. coli, ceftazidime-resistant $K$. pneumoniae, oxacillinresistant Staphylococcus aureus, ceftazidime-resistant Pseudomonas aeruginosa, and ciprofloxacin-resistant $P$. aeruginosa $(\rho=-1, P<0.01)$.

Conclusions. The trend analysis presented in this study is the baseline for establishing a national epidemiological surveillance subsystem. The trends observed reveal that bacterial resistance to antimicrobial drugs in hospitals in Colombia is a dynamic phenomenon, with evidence of the emergence of vancomycin-resistant E. faecium and imipenem-resistant K. pneumoniae phenotypes in the hospitals.

Key words Anti-bacterial agents; drug resistance, bacterial; epidemiologic surveillance; hospitals; Colombia. 